\title{
EXISTENCE AND OSCILLATION THEOREMS FOR NON-LINEAR DIFFERENTIAL SYSTEMS OF THE SECOND ORDER*
}

\author{
BY
}

WILLIAM M. WHYBURN

\section{Introduction}

Boundary value problems for second order linear differential systems ave been investigated extensively by many authors of the past century. $t$ is the object of the present paper to consider non-linear equations of the econd order and to establish existence and oscillation theorems for these equations together with boundary conditions of the two-point type. The coefficients of the differential equations are taken as summable functions of the independent variable and all integrals in the paper are taken in the sense of Lebesgue. The quantities used are confined to the real domain.

\section{EXISTENCE THEOREMS}

The following two theorems are special cases of general existence theorems that have recently been established by the author. $\dagger$

Theorem I. Given the differential system

$$
\begin{aligned}
d y_{i} / d x & =\sum_{j=1}^{j=n} A_{i j}\left(x, y_{1}, \cdots, y_{n}\right) y_{i} & \\
y_{i}(c) & =a_{i} & (i=1,2, \cdots, n),
\end{aligned}
$$

where the functions $A_{i j}$ are single-valued and continuous in $\left(y_{1}, \cdots, y_{n}\right)$ for each fixed $x$ on $X: a \leqq x \leqq b$, summable (Lebesgue) in $x$ on $X$ for fixed $\left(y_{1}, \cdots, y_{n}\right)$, and bounded numerically for all $x$ on $X$ and for all $\left(y_{1}, \cdots, y_{n}\right)$ by a function $M(x)$ that is summable on $X$, and where $x=c$ is any fixed point of $X$ and the quantities $a_{1}, \cdots, a_{n}$ are constants, then there exists a unique solution $\ddagger$ of system (1), (2), provided that there exists a summable function $N(x)$ on $X$ such that

* Presented to the Society, September 7, 1927; received by the editors in August, 1927.

$\dagger$ W. M. Whyburn, On the fundamental existence theorems for differential systems, to appear in Annals of Mathematics for December, 1928.

$\ddagger$ By a solution of this system we mean a set of absolutely continuous functions on $X$ that satisfies (2) and satisfies (1) almost everywhere on $X$. 


$$
\begin{aligned}
\left|A_{i j}\left(x, y_{1}, \cdots, y_{n}\right)-A_{i j}\left(x, z_{1}, \cdots, z_{n}\right)\right| \leqq N(x) & \sum_{r=1}^{r=n}\left|y_{r}-z_{r}\right| \\
& (i, j=1, \cdots, n)
\end{aligned}
$$

for all $\left(y_{1}, \cdots, y_{n}\right)$ and $\left(z_{1}, \cdots, z_{n}\right)$ and for every $x$ on $X$.

ThEOREM II. For the system

$$
\begin{aligned}
& d y_{i} / d x=\sum_{j=1}^{j=n} A_{i j}\left(x, y_{1}, \cdots, y_{n} ; \lambda\right) y_{j} \quad(i=1, \cdots, n), \\
& y_{i}(c, \lambda)=a_{i}(\lambda)
\end{aligned}
$$

where $a_{1}, \cdots, a_{n}$ are continuous functions of $\lambda$ on $\bar{L}: \bar{L}_{1} \leqq \lambda \leqq \bar{L}_{2} ;$ the functions $A_{i j}$ are summable in $x$ on $X$ for each fixed $\left(y_{1}, \cdots, y_{n}\right)$ and each fixed $\lambda$ on $\bar{L}$, continuous in $\left(y_{1}, \cdots, y_{n}, \lambda\right)$ for all $\left(y_{1}, \cdots, y_{n}\right)$ and all $\lambda$ on $\bar{L}$ when $x$ has a fixed value on $X$, bounded numerically by a function $M(x)$ that is summable on $X$, and finally there is a summable function $N(x)$ on $X$ such that for all $\lambda$ on $\bar{L}$ and for every $\left(y_{1}, \cdots, y_{n}\right)$ and $\left(z_{1}, \cdots, z_{n}\right)$

$$
\begin{array}{r}
\left|A_{i j}\left(x, y_{1}, \cdots, y_{n} ; \lambda\right)-A_{i j}\left(x, z_{1}, \cdots, z_{n} ; \lambda\right)\right| \leqq N(x) \sum_{r=1}^{r=n}\left|y_{r}-z_{r}\right| \\
(i, j=1, \cdots, n),
\end{array}
$$

there exists on $X \bar{L}$ a unique set $y_{1}(x, \lambda), \cdots, y_{n}(x, \lambda)$ of continuous* functions such that for each fixed $\lambda$ on $\bar{L}$ these functions are absolutely continuous in $x$ on $X$ and satisfy (3) almost everywhere on $X$, and such that the set of functions satisfies (4) everywhere on $\bar{L}$.

COROLLARY. If the hypotheses of Theorem II are met for every closed subinterval of the open $\lambda$-interval $L: L_{1}<\lambda<L_{2}$, then the conclusions of that theorem are valid for the domain $X L$.

\section{THE SECOND-ORDER SYSTEM}

Consider the system

$$
d y / d x=K(x, y, z ; \lambda) z, \quad d z / d x=G(x, y, z ; \lambda) y,
$$

where $K$ and $G$ satisfy the hypotheses of Theorem II and its corollary. Let $y(x, \lambda), z(x, \lambda)$ denote the unique solution of (5) that satisfies the conditions $y(a, \lambda)=0, z(a, \lambda)=1$, for all values of $\lambda$ on $L$.

To system (5) apply the transformation $\dagger$

* Cf. Carathéodory, Vorlesungen über reele Funktionen, Berlin, 1918, p. 678.

$\dagger$ The use of this transformation in the study of boundary value problems was suggested to me by Professor H. J. Ettlinger. 
This yields

$$
\begin{aligned}
& y(x, \lambda)=u(x, \lambda) \sin v(x, \lambda), \\
& z(x, \lambda)=u(x, \lambda) \cos v(x, \lambda) .
\end{aligned}
$$

$$
\begin{aligned}
& d v / d x=K \cos ^{2} v-G \sin ^{2} v, \\
& d u / d x=\frac{u[K+G]}{2} \sin 2 v .
\end{aligned}
$$

Carathéodory's existence theorem* yields at least one solution of (6) that satisfies $u(c, \lambda)=a_{1}, v(c, \lambda)=a_{2}$, where $x=c$ is a point of $X$ and $a_{1}$ and $a_{2}$ are continuous functions of $\lambda$ on $L$. Suppose that for some fixed $\lambda$ on $L$ there is more than one solution of (6) and these initial conditions. Let $u_{1}, v_{1}$ and $u_{2}, v_{2}$ be two such solutions and suppose for definiteness that they are not identical on $C: c \leqq x \leqq b$. Obviously the case where these solutions are identical on $C$ but differ on $a \leqq x \leqq c$ can be obtained from the present case by modifying the notation slightly and inserting additional absolute value signs in the inequalities. Let $H$ be a constant that is greater than any of the continuous functions $1,\left|u_{1}\right|,\left|v_{1}\right|,\left|u_{2}\right|,\left|v_{2}\right|$, on $X$. Let $K_{1}=K(x$, $\left.u_{1} \sin v_{1}, u_{1} \cos v_{1}\right), G_{1}=G\left(x, u_{1} \sin v_{1}, u_{1} \cos v_{1}\right), K_{2}=K\left(x, u_{2} \sin v_{2}, u_{2} \cos v_{2}\right)$, and $G_{2}=G\left(x, u_{2} \sin v_{2}, u_{2} \cos v_{2}\right)$. From (6) we have

$$
\begin{aligned}
v_{1}-v_{2} & =\int_{c}^{x}\left[K_{1} \cos ^{2} v_{1}-K_{2} \cos ^{2} v_{2}-\left(G_{1} \sin ^{2} v_{1}-G_{2} \sin ^{2} v_{2}\right)\right] d t \\
\left|v_{1}-v_{2}\right| \leqq & \int_{c}^{x}\left[\left|K_{1}-K_{2}\right|+\left|G_{1}-G_{2}\right|\right. \\
& \left.\quad+\left(\left|K_{2}\right|+\left|G_{2}\right|\right)\left(\left|\sin ^{2} v_{1}-\sin ^{2} v\right|\right)\right] d t \\
& \leqq 2 \int_{c}^{x}\left(N\left[\left|u_{1} \sin v_{1}-u_{2} \sin v_{2}\right|+\left|u_{1} \cos v_{1}-u_{2} \cos v_{2}\right|\right]\right. \\
& \left.+2 M\left|\sin v_{1}-\sin v_{2}\right|\right) d t
\end{aligned}
$$

since $\left|\sin \left[\left(v_{1}-v_{2}\right) / 2\right]\right| \leqq\left|\left(v_{1}-v_{2}\right) / 2\right|$ and $H$ is greater than unity. Similarly

$$
\left|u_{1}-u_{2}\right|<4 \int_{c}^{x} H(N H+M)\left(\left|u_{1}-u_{2}\right|+\left|v_{1}-v_{2}\right|\right) d t .
$$

The foregoing inequalities are valid for each $x$ on $C$.

* Loc. cit., p. 672 ff. This theorem does not apply to give uniqueness. 
Let $v(x)=\left|u_{1}-u_{2}\right|+\left|v_{1}-v_{2}\right|$ and add the above inequaltities to get

$$
\begin{aligned}
& v(x)<8 \int_{c}^{x} H[H N(t)+M(t)] v(t) d t, \\
& v(x)<8 \int_{d}^{x} H[H N(t)+M(t)] v(t) d t,
\end{aligned}
$$

where $x=d$ is the last point of $C$ such that $v(x) \equiv 0$ on $c \leqq x \leqq d$. Let $x_{1}$ be any point of $d<x \leqq b$ and let $x_{2}$ be a point of $d \leqq x \leqq x_{1}$ at which the continuous function $v(x)$ takes on its maximum value for that interval. From (7)

$$
v\left(x_{2}\right)<v\left(x_{2}\right) \int_{d}^{x_{2}} 8 H[H N+M] d t \leqq v\left(x_{2}\right) \int_{d}^{x_{1}} 8 H[H N+M] d t
$$

and since $v\left(x_{2}\right)>0$, we may divide by it to get

$$
\int_{d}^{x_{1}} 8 H[H N(t)+M(t)] d t>1 .
$$

This inequality must hold for every $x_{1}$ on $d<x_{1} \leqq b$ which evidently is impossible. Hence we have established the following theorem:

Theorem III. There exists a unique pair of functions $u(x, \lambda), v(x, \lambda)$ each of which is continuous in $(x, \lambda)$ on $X L$, absolutely continuous in $x$ on $X$ for each fixed $\lambda$ on $L$, and such that the pair satisfies the conditions $u(c, \lambda)=a_{1}(\lambda)$, $v(c, \lambda)=a_{2}(\lambda)$ for every $\lambda$ on $L$ and satisfies system (6) almost everywhere on $X$ for each fixed $\lambda$ on $L$.

TheOREM IV. If $u(x, \lambda), v(x, \lambda)$ is the solution of $(6)$ such that $u(a, \lambda) \equiv 1$, $v(a, \lambda) \equiv 0$, then $u(x, \lambda)$ does not vanish on $X L$.

Assume that $u(c, p)=0$. By Theorem III there exists a unique solution $U, V$ of (6) on $a \leqq x \leqq c$ satisfying the conditions $U(c, p)=0, V(c, p)=v(c, p)$. The method of successive approximations yields a solution of this system for which $u(x, p) \equiv 0$ on $a \leqq x \leqq c$ and hence this solution must be identical with the one that we are considering. This, however, contradicts the fact that $u(a, p)=1$ and yields our theorem.

In the discussion which follows we will assume that $K(x, y, z ; \lambda)$ and $-G(x, y, z ; \lambda)$ are positive for all values of $x$ and $\lambda$ on $X L$ and for all values of $y$ and $z$. It follows immediately from (6) that for each fixed value of $\lambda$ on $L$ the function $v$ is an increasing function of $x$ on $X$. This gives a one-to-one continuous correspondence between the points of $v(a) \leqq v \leqq v(b)$ and the points of $X$. Furthermore, this correspondence preserves order so that if $x_{1}$ and $x_{2}$ are any two points of $X$ such that $x_{1}<x_{2}$, then $v\left(x_{1}\right)<v\left(x_{2}\right)$. Since 
$u(x, \lambda)>0$ on $X L$ the zeros of $y$ coincide with the zeros of $\sin v$ and the zeros of $z$ coincide with those of $\cos v$. If for a fixed $\lambda$ on $L,(2 m-1) \pi / 2 \leqq v(b) \leqq m \pi$, where $m$ is a positive integer, then $y$ will have zeros for $v(x)=i \pi$ while $z$ has zeros for $v(x)=(2 i+1) \pi / 2$, where $i=1,2, \cdots, m-1$. It follows immediately from the correspondence between $v$ and $x$ that the zeros of $y$ and $z$ separate each other on $X$.

Condition A. There exists a value $\lambda=p$ on $L$ such that if $q$ is any fixed value of $\lambda$ on $L_{1}<\lambda \leqq p$ and $M$ and $\bar{M}$, respectively, denote the upper bounds of $K$ and $-G$ for this value of $\lambda$, then $M+\bar{M}<\pi /(b-a)$.

Condition B. There exists a subinterval $D: c \leqq x \leqq d$ of $X$ such that if $m(\lambda)$ denotes the smaller of the lower bounds of $K$ and $-G$ on $D$ for each fixed $\lambda$, then $\lim _{\lambda \rightarrow L_{2}} m(\lambda)=+\infty$.

Condition A insures that for every $\lambda$ on $L_{1}<\lambda \leqq p, v(b, \lambda)<\pi$ and consequently that $y(x, \lambda)$ does not vanish on $a<x \leqq b$ for fixed values of $\lambda$ on $L_{1}<\lambda \leqq p$. This follows since $v(b, \lambda)=\int_{a}\left(K \cos ^{2} v-G \sin ^{2} v\right) d t \leqq \int_{a}^{b}[M+\bar{M}] d t$ $<(b-a) \pi /(b-a)$.

Condition B insures that $\lim _{\lambda \rightarrow L_{2}} v(b, \lambda)=+\infty$, since $v(b, \lambda) \geqq \int_{c}^{d}\left(K \cos ^{2} v\right.$ $\left.-G \sin ^{2} v\right) d t>m(\lambda)(d-c)$ and $\lim _{\lambda \rightarrow L_{2}} m(\lambda)=+\infty$.

THEOREM V. If $K$ and $-G$ are positive on $X L$ and for every $y$ and $z$ and if Conditions $\mathrm{A}$ and $\mathrm{B}$ are satisfied, then there exists an infinite collection, $k_{1}, k_{2}, \cdots$, of sets of characteristic numbers* for the system (5), (8) such that if $p_{i}$ is any number in $k_{i}$, then $y\left(x, p_{i}\right)$ and $z\left(x, p_{i}\right)$ each have exactly $i$ zeros on $a<x \leqq b$, and finally for each index $i$ there exists an index $j$ such that for every $n$ greater than $j$, every number in $k_{n}$ is greater than every number in $k_{i}$. System (8) is

$$
y(a, \lambda)=0, \quad y(b, \lambda)=0 .
$$

As $\lambda$ increases from a value between $L_{1}$ and $p$ to $L_{2}, v(b, \lambda)$ increases from a value less than $\pi$ to $+\infty$. If $i$ is any positive integer, it follows from the continuity of $v(b, \lambda)$ in $\lambda$ that for at least one value of $\lambda$ on $L, v(b, \lambda)=i \pi$. Let $k_{i}$ be the totality of values of $\lambda$ on $L$ for which $v(b, \lambda)=i \pi$ and let $p_{i}$ be any value in $k_{i}$; then since $v\left(x, p_{i}\right)$ is an increasing, continuous function of $x$ on $X$, it follows that for one and only one value of $x$ on $X, v\left(x, p_{i}\right)=j \pi$, $j=1,2, \cdots, i$. Also, since $\lim _{\lambda \rightarrow L_{2}} v(b, \lambda)=+\infty$, we can determine $q_{i}$ such that for every $\lambda$ on $q_{i} \leqq \lambda<L_{2}, v(b, \lambda)>i \pi$ and hence no values of $k_{i}$ can lie on $q_{i} \leqq \lambda<L_{2}$. We can also determine $j$ such that $v(b, \lambda)<j \pi$ on $p \leqq \lambda \leqq q_{i}$

* By a characteristic number we mean a value of $\lambda$ for which the system has a non-indentically vanishing solution. 
and hence no values in $k_{j}, k_{j+1}, \cdots$ lie on $p \leqq \lambda \leqq q_{i}$. Hence for any $n>j$, every number in $k_{n}$ is greater than every number in $k_{i}$. This completes the proof of Theorem $\mathrm{V}$.

A consideration of the values of $\lambda$ for which $v(b, \lambda)=(2 i-1) \pi / 2$ yields the following theorem:

THEOREM VI. Under the hypotheses of Theorem V there exists an infinite collection of sets, $h_{2}, h_{3}, \cdots$, of characteristic numbers for the system (5) $y(a, \lambda)=0, z(b, \lambda)=0$ such that if $p_{i}$ is any value in $h_{i}, y\left(x, p_{i}\right)$ and $z\left(x, p_{i}\right)$ each have exactly $i$ zeros on $X$ and such that for each $i$ there exists an index $j$ such that for every $n$ greater than $j$, every number in $h_{n}$ is greater than every number in $h_{i}$.

By letting $y=u(x, \lambda) \cos v(x, \lambda), z=u(x, \lambda) \sin v(x, \lambda)$ and repeating the foregoing discussion, we get existence and oscillation theorems of the above type for the system (5) together with the boundary conditions $z(a, \lambda)=0$, $y(b, \lambda)=0$ and with the boundary conditions $z(a, \lambda)=0, z(b, \lambda)=0$.

We close this section with an example of a non-linear system to which all of the foregoing theory applies. Consider the system

$$
\begin{aligned}
d y / d x & =\lambda\left(\sin ^{2} y+2\right) z / x^{2 / 3}, & X: 1 \leqq x \leqq b, \\
d z / d x & =-\lambda\left(2 \cos ^{2} z+3\right) y / x^{4 / 5}, & L: 0<\lambda<+\infty,
\end{aligned}
$$

together with boundary conditions of any of the four types considered in this section. One easily sees that all of the hypotheses of the foregoing theorems are met by this system and hence Theorems II, III, IV, V, VI are valid. Many other examples can be constructed to illustrate the systems covered by this treatment.

\section{The LINEAR SYSTEM}

The case where $K$ and $G$ do not depend on $y$ and $z$ is of especial interest in that it gives a new treatment of boundary value problems of the Sturmian type.* The linear case appears as a special case of all of the foregoing treatment and many of the hypotheses are automatically satisfied for this case. It is noted that in the present treatment all of the conditions on $K$ and $-G$ are symmetric, which is to be expected when one notes the manner in which these coefficients enter into the system. Examples can be given of systems where conditions of the above type are highly desirable. $\dagger$

* Cf. Bocher, Leçons sur les Méthodes de Sturm, Paris, 1917, p. 66ff.

$\dagger$ Such an example arises in the minimum surface of revolution problem of the calculus of variations. Conditions for non-oscillation are desired for the system $y^{\prime}=\left(1+\lambda^{2}\right)^{1 / 2} \cosh \left[x\left(1+\lambda^{2}\right)^{1 / 2}\right.$ $\left.+\sinh ^{-1} \lambda\right] z, z^{\prime}=-\left(1+\lambda^{2}\right)^{1 / 2} \operatorname{sech}\left[x\left(1+\lambda^{2}\right)^{1 / 2}+\sinh ^{-1} \lambda\right] y$. The usual conditions of $K$ and $G$ being positive cannot be satisfied for real values of $x$ and $\lambda$ while Condition $A$ of the above theory can be applied to obtain the desired information. 
TheOREM VII. If for each fixed $x_{1}$ on $a<x \leqq b, K$ and $-G$ are nondecreasing functions of $\lambda$ on $L$ and there exists a sub-interval $D\left(x_{1}\right)$ of $a<x \leqq x_{1}$ almost everywhere on which either $\delta K$ or $-\delta G$ is positive, where $\delta f$ is the increment in $f$ due to a positive increment $\delta \lambda$ in $\lambda$, then $v\left(x_{1}, \lambda\right)$ is an increasing function of $\lambda_{0}$ on $L$.

Choose $\delta \lambda$ so that $\lambda+\delta \lambda$ lies on $L$. From (6) $d(\delta v) / d x=\left[\delta K \cos ^{2}(v+\delta v)-\delta G \sin ^{2}(v+\delta v)\right]-(K+G) \sin (2 v+\delta v) \sin \delta v$. Using the continuity of $v$ in $(x, \lambda)$ and restricting the size of $\delta \lambda$, we may solve this equation to get

$$
\delta v\left(x_{1}, \lambda\right)=e^{-\omega}\left\{\int_{a}^{x_{1}}\left[\delta K \cos ^{2}(v+\delta v)-\delta G \sin ^{2}(v+\delta v)\right] e^{\tau} d t\right\}
$$

where

$$
\begin{gathered}
\omega=\int_{a}^{x_{1}}\{[K+G](\sin (2 v+\delta v))(\sin \delta v) / \delta v\} d t, \\
\tau=\int_{a}^{t}\{[K+G](\sin (2 v+\delta v))(\sin \delta v) / \delta v\} d s,
\end{gathered}
$$

from which we conclude immediately that $\delta v\left(x_{1}, \lambda\right)>0$.

Under the hypotheses of Theorem VII there exists a one-to-one, orderpreserving correspondence between the points of the $v$-interval and those of $L$ for each fixed $x$ on $a<x \leqq b$. This correspondence enables us to state that $k^{*}$ and $h_{i}$ of Theorems V and VI contain one and only one value of $\lambda$ and that these values are ordered in the following fashion:

$$
L_{1}<k_{1}<h_{2}<k_{2}<h_{3}<\cdots<h_{i}<k_{i}<\cdots<L_{2} .
$$

A transformation that was used by the author in another paper* can be used to show that $\phi(x, \lambda)=\alpha(x, \lambda) y-\beta(x, \lambda) z$ and $\psi(x, \lambda)=\gamma(x, \lambda) y-\delta(x, \lambda) z$, where $\alpha, \beta, \delta, \gamma$ have simple properties as functions of $x$ and $\lambda$, satisfy equations of the type of (5) ( $K$ and $G$ are linear). Hence the foregoing treatment can be used to prove existence and oscillation theorems where the boundary conditions are $\phi(a, \lambda)=\phi(b, \lambda)=0$.

* These Transactions, vol. 30, pp. 630-640.

UNIVERSITY OF TEXAS, Austan, Texas 